\title{
An IMRT/VMAT Technique for Nonsmall Cell Lung Cancer
}

\author{
Nan Zhao, Ruijie Yang, Junjie Wang, Xile Zhang, and Jinna Li \\ Department of Radiation Oncology, Peking University Third Hospital, Beijing 100191, China \\ Correspondence should be addressed to Ruijie Yang; ruijyang@yahoo.com
}

Received 9 May 2015; Revised 13 July 2015; Accepted 21 July 2015

Academic Editor: Jun Deng

Copyright (C) 2015 Nan Zhao et al. This is an open access article distributed under the Creative Commons Attribution License, which permits unrestricted use, distribution, and reproduction in any medium, provided the original work is properly cited.

\begin{abstract}
The study is to investigate a Hybrid IMRT/VMAT technique which combines intensity modulated radiation therapy (IMRT) and volumetric modulated arc therapy (VMAT) for the treatment of nonsmall cell lung cancer (NSCLC). Two partial arcs VMAT, 5-field IMRT, and hybrid plans were created for 15 patients with NSCLC. The hybrid plans were combination of 2 partial arcs VMAT and 5-field IMRT. The dose distribution of planning target volume (PTV) and organs at risk (OARs) for hybrid technique was compared with IMRT and VMAT. The monitor units (MUs) and treatment delivery time were also evaluated. Hybrid technique significantly improved the target conformity and homogeneity compared with IMRT and VMAT. The mean delivery time of IMRT, VMAT, and hybrid plans was $280 \mathrm{~s}, 114 \mathrm{~s}$, and $327 \mathrm{~s}$, respectively. The mean MUs needed for IMRT, VMAT, and hybrid plans were 933, 512, and 737, respectively. Hybrid technique reduced $V_{5}, V_{10}, V_{30}$, and MLD of normal lung compared with VMAT and spared the OARs better with fewer MUs with the cost of a little higher $V_{5}, V_{10}$, and mean lung dose (MLD) of normal lung compared with IMRT. Hybrid IMRT/VMAT can be a viable radiotherapy technique with better plan quality.
\end{abstract}

\section{Introduction}

Treatment of nonsmall cell lung cancer (NSCLC) remains one of the major challenges for radiotherapy. Three-dimensional conformal radiotherapy (3D-CRT) has proved to be a promising treatment method for NSCLC allowing higher doses to be delivered to the target by improved shaping of radiation portals and conformal avoidance of normal structures compared with the conventional radiotherapy [1]. Compared to 3DCRT, intensity modulated radiation therapy (IMRT) further significantly improved the dose conformity and sparing of organs at risk [2]. However, the longer treatment time in IMRT could increase the discomfort of the patients during the treatment, and more MUs could increase the incidence of secondary radiation-induced cancer $[3,4]$. Volumetric modulated arc therapy (VMAT) provided more conformal target coverage and better sparing of organs at risk (OARs), with shorter treatment delivery time and fewer MUs than IMRT in treating cancers of different sites [5-9]. However, a larger volume of lung receiving lower dose $\left(V_{5}\right.$ and $\left.V_{10}\right)$ in VMAT has been reported [10]. Dose volume histogram parameters of $V_{5}[11-14]$ and $V_{10}[12,14,15]$ have been showed to be the predictors of the radiation pneumonitis.
The aim of this study is to investigate a radiotherapy technique we call Hybrid IMRT/VMAT for nonsmall cell lung cancer treatments. The dosimetric quality and delivery efficiency of the Hybrid IMRT/VMAT technique were evaluated by comparing with IMRT and VMAT for 15 nonsmall cell lung cancer patients.

\section{Methods and Materials}

2.1. Patients' Characteristics. Fifteen NSCLC patients who underwent radiotherapy from January 2012 to April 2013 in our hospital were retrospectively selected for this study.

2.2. Delineation of Target Volumes and Critical Structures. The patients underwent four-dimensional computed tomography (4D-CT) (Brilliance Big Bore, Philips Medical Systems, Cleveland, USA) scanning in $5 \mathrm{~mm}$ slice thickness, $0.5 \mathrm{sec}$ onds of scan time per rotation during normal breathing in supine arm-up position. The gross tumor volume (GTV) was defined as the visualization of any gross tumor and lymph nodes involved $(>1 \mathrm{~cm}$ on $\mathrm{CT})$. An internal target volume (ITV) was obtained as a union of the GTVs from all respiratory motion phases. The CTV was defined as the potential 
TABLE 1: Treatment planning objectives used for Hybrid IMRT/ VMAT, IMRT, and VMAT plans.

\begin{tabular}{lcc}
\hline \multirow{2}{*}{ PTV } & $D_{98 \%}$ & $>62.7 \mathrm{~Gy}$ \\
& $D_{2 \%}$ & $<72.6 \mathrm{~Gy}$ \\
\hline \multirow{4}{*}{ Normal lung } & ${ }^{*} V_{5}$ & $<60 \%$ \\
& ${ }^{*} V_{10}$ & $<40 \%$ \\
& ${ }^{*} V_{20}$ & $<30 \%$ \\
& ${ }^{*} V_{30}$ & $<20 \%$ \\
\hline Spinal cord & Mean dose & $<16 \mathrm{~Gy}$ \\
\hline \multirow{2}{*}{ Esophagus } & Max dose & $<50 \mathrm{~Gy}$ \\
\hline \multirow{3}{*}{ Heart } & Max dose & $<66 \mathrm{~Gy}$ \\
& Mean dose & $<34 \mathrm{~Gy}$ \\
\hline
\end{tabular}

PTV is planning target volume.

${ }^{*} V_{N}$ is percentage volume of OARs receiving at least $N$ Gy of radiation dose.

harboring microscopic disease. The PTV was created by expanding the CTV by $0.5 \mathrm{~cm}$. The OARs delineated included the double lungs, normal lung, spinal cord, esophagus, and heart. We defined the double lungs minus GTV as normal lung. The spinal cord and the esophagus were contoured starting at least $2 \mathrm{~cm}$ above the superior extent of the PTV and continuing on every CT slice to at least $2 \mathrm{~cm}$ below the inferior extent of the PTV. No margins were added to the organs at risk.

2.3. Treatment Planning. Hybrid IMRT/VMAT, IMRT, and VMAT plans were designed for each patient. The prescribed dose to the PTV was $66 \mathrm{~Gy}$ in 33 fractions. The plans were normalized to cover $95 \%$ of the PTV with $100 \%$ of the prescribed dose. The optimization objectives and constraints shown in Table 1 were the same for the three techniques. Eclipse 10.0 (Varian, Palo Alto, CA) treatment planning system was used for all treatment planning, utilizing $6 \mathrm{MV}$ photon beams generated from Varian Trilogy linac equipped with a 120 leaf Millennium Multileaf Collimator (MLC).

2.4. IMRT. The beam angles of IMRT were initially optimized by the beam angle optimization algorithm (Varian Eclipse 10.0); a set of initial optimization objectives were loaded into the treatment planning system. The number of the fields was confined to five. Some beam angles were adjusted according to the experience of the dosimetrists, if the results of the beam angle optimization did not satisfy the dosimetric criteria. The plans were iteratively optimized to obtain the optimal PTV coverage and OARs sparing. After inverse planning, the leaf sequences using sliding window technique were generated for IMRT plans.

2.5. VMAT. All VMAT plans were generated using 2 partial arcs. The collimator angle varied between $0^{\circ}$ and $90^{\circ}$ according to the shape of the target while minimizing the leakage, tongue, and groove effects. Other planning parameters were
MLC motion speed 0 to $2.5 \mathrm{~cm} / \mathrm{s}$, gantry rotation speed 0.5 to $4.8 \mathrm{degrees} / \mathrm{s}$, and dose rate 0 to $600 \mathrm{MU} / \mathrm{min}$.

2.6. Hybrid IMRT/VMAT. The Hybrid IMRT/VMAT technique integrates IMRT and VMAT. The IMRT part consists of a 5-field IMRT plan (Hybrid-IMRT), which contributes half of the total prescribed dose, while the VMAT parts consist of a 2 partial arcs VMAT plan (Hybrid-VMAT) which was optimized with the IMRT plan as a base plan, to deliver the other half of the prescribed dose.

2.7. Dosimetric Evaluation. The dosimetric quality of the Hybrid IMRT/VMAT plans was evaluated by comparison with IMRT and VMAT. To evaluate the dose distribution of the target, we calculated the minimal dose delivered to the $98 \%$ of the target volume $\left(D_{98 \%}\right)$, the maximum dose delivered to the $2 \%$ of the target volume $\left(D_{2 \%}\right)$, the median absorbed dose delivered to the $50 \%$ of the target volume $\left(D_{50 \%}\right)$, conformation number $(\mathrm{CN})$, and homogeneity index (HI) according to the ICRU report 83 [16]. All parameters were computed on the basis of the DVH. The CN was defined using the equation [11]

$$
\mathrm{CN}=\frac{\mathrm{TV}_{\mathrm{RI}}}{\mathrm{TV}} \times \frac{\mathrm{TV}_{\mathrm{RI}}}{V_{\mathrm{RI}}},
$$

where $\mathrm{CN}$ is conformation number, $\mathrm{TV}_{\mathrm{RI}}$ is target volume covered by the reference isodose, TV is target volume, and $V_{\mathrm{RI}}$ is volume of the reference isodose. The $\mathrm{CN}$ ranged from 0 to 1 , where 1 was the ideal value. A larger $\mathrm{CN}$ indicated a smaller volume of the prescription dose delivered outside the PTV. The HI was defined using the equation [16]

$$
\mathrm{HI}=\frac{D_{2 \%}-D_{98 \%}}{D_{50 \%}} .
$$

An $\mathrm{HI}$ of 0 indicated that the dose distribution was almost homogenous. A larger $\mathrm{HI}$ indicated a greater dose exceeding the prescribed dose and/or a larger volume of the target receiving too small dose. The evaluation criteria of OARs were defined basically according to RTOG 1106 protocols. $V_{5}, V_{10}, V_{20}, V_{30}$, and mean lung dose (MLD) values were recorded and compared for normal lung, as well as the maximum dose of the spinal cord, the mean and maximum dose of the esophagus, the $D_{2 \%}, V_{40}, V_{60}$, and mean dose of the heart.

2.8. Treatment Delivery Time and MUs. The Hybrid IMRT/ VMAT, IMRT, and VMAT plans for 15 patients were delivered to a solid water phantom (Multicube Phantom, IBA, Germany) on the Trilogy linear accelerator. The treatment delivery time and MUs were recorded and evaluated. The treatment delivery time was defined as the time from first beam on until last beam off.

2.9. Dosimetric Evaluation Stratified by Target Volume. In order to investigate the target volume effect on the selection of the optimal technique, we separated the 15 patients into two groups according to the volumes of the PTVs, 8 patients with 

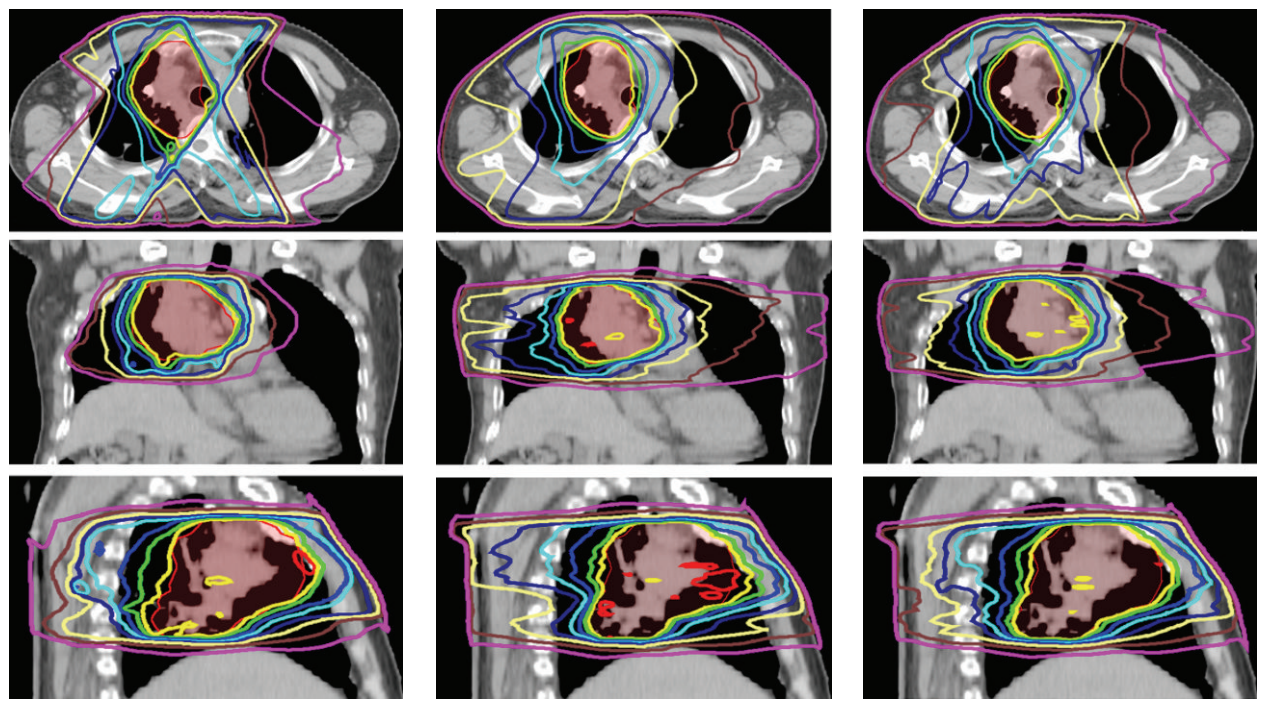

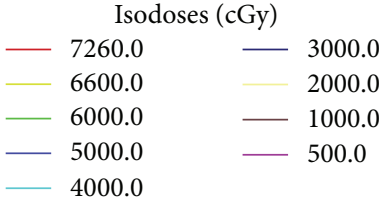

(a)

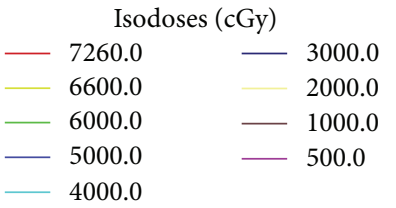

(b)

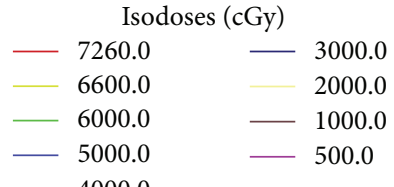

(c)

FIGURE 1: Representative axial, coronal, and sagittal computed tomography slices showing isodose distribution for (a) IMRT, (b) VMAT, and (c) Hybrid IMRT/VMAT. Planning target volume (PTV) shown in red.

the PTV volumes smaller and 7 patients larger than the mean volume of the PTVs $\left(416.1 \mathrm{~cm}^{3}\right)$. The dose distribution of planning target volume (PTV) and organs at risk (OARs) for Hybrid IMRT/VMAT was compared with IMRT and VMAT for two groups separately.

2.10. Statistical Analysis. Paired two tailed $t$-test was used to compare the three techniques. Statistical analysis was performed using the SPSS (version 13.0, Chicago, IL) for Windows. Differences were reported to be statistically significant at $p<0.05$.

\section{Results}

The mean volume of the PTV was $416.1 \mathrm{~cm}^{3}\left(173.4 \mathrm{~cm}^{3}\right.$ to $887.0 \mathrm{~cm}^{3}$ ). For all 15 cases, all the plans were clinically acceptable in terms of target coverage, with at least 98\% PTV receiving $95 \%$ of the prescribed dose. The typical isodose distribution and DVH comparison were given in Figures 1 and 2 for a patient with stage IIIB nonsmall cell lung cancer. The PTV was $414.0 \mathrm{~cm}^{3}$. The lesions were located in the right hilus pulmonis and the upper lobe of the right lung. The beams obtained by the beam angle optimization for IMRT are $39^{\circ}, 150^{\circ}, 210^{\circ}, 306^{\circ}$, and $342^{\circ}$. Two partial arcs of $0^{\circ} \sim 181^{\circ}$ and $181^{\circ} \sim 0^{\circ}$ were used for VMAT.

3.1. Target Coverage. The data for PTV coverage and OARs sparing of IMRT, VMAT, and Hybrid IMRT/VMAT plans

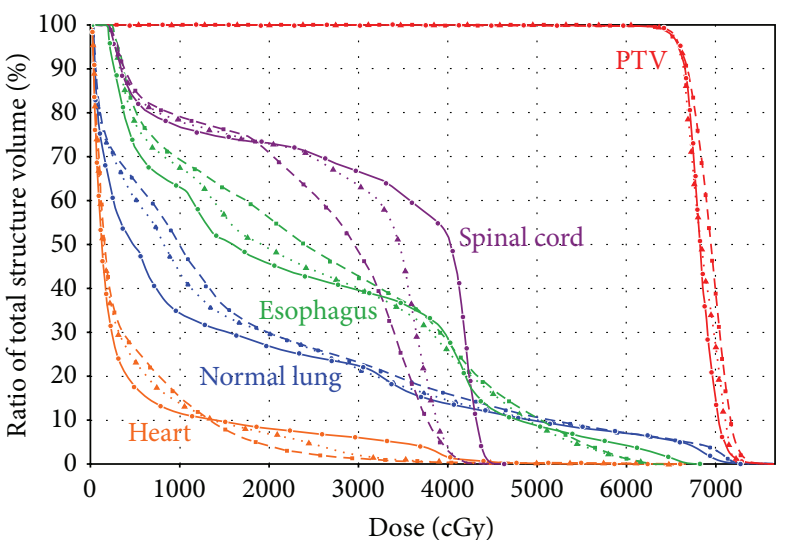

FIGURE 2: Representative dose volume histogram for IMRT, VMAT, and Hybrid IMRT/VMAT. The curves of IMRT, VMAT, and Hybrid IMRT/VMAT are indicated in solid lines, dashed lines, and dotted lines, respectively.

were summarized in Tables 2 and 3. Hybrid IMRT/VMAT significantly improved the target conformity compared with IMRT and VMAT. The mean CN was $0.79,0.86$, and 0.88 for IMRT, VMAT, and hybrid plans, respectively. Hybrid IMRT/VMAT also significantly improved the PTV dose homogeneity compared with IMRT (9.8 versus 11.3; $p<0.05$ ) and VMAT ( 9.8 versus 12.6; $p<0.05$ ). Compared with IMRT, VMAT also improved the dose conformity. 
TABLE 2: The data for PTV coverage for IMRT, VMAT, and Hybrid IMRT/VMAT plans.

\begin{tabular}{lcccccc}
\hline & IMRT & VMAT & Hybrid & IMRT versus VMAT & IMRT versus Hybrid & VMAT versus Hybrid \\
& Mean \pm vD & Mean \pm SD & Mean \pm SD & value & & value \\
\hline PTV & & & & & $<0.05$ & $<0.05$ \\
$D_{98 \%}(\mathrm{~Gy})$ & $64.6 \pm 0.5$ & $64.6 \pm 0.5$ & $65.0 \pm 0.3$ & $>0.05$ & $<0.05$ & $<0.05$ \\
$D_{2 \%}(\mathrm{~Gy})$ & $72.6 \pm 1.7$ & $73.3 \pm 1.7$ & $71.5 \pm 1.0$ & $>0.05$ & $<0.05$ & $<0.05$ \\
$\mathrm{CN}$ & $0.79 \pm 0.05$ & $0.86 \pm 0.04$ & $0.88 \pm 0.03$ & $<0.05$ & $<0.05$ & $<0.05$ \\
$\mathrm{HI}(\%)$ & $11.3 \pm 0.7$ & $12.6 \pm 0.6$ & $9.8 \pm 0.3$ & $<0.05$ & & \\
\hline
\end{tabular}

PTV is planning target volume, IMRT is intensity modulated radiation therapy, VMAT is volumetric modulated arc therapy, CN is conformation number, and $\mathrm{HI}$ is homogeneity index.

TABLE 3: The data for OARs sparing for IMRT, VMAT, and Hybrid IMRT/VMAT plans.

\begin{tabular}{|c|c|c|c|c|c|c|}
\hline & $\begin{array}{c}\text { IMRT } \\
\text { Mean } \pm \text { SD }\end{array}$ & $\begin{array}{c}\text { VMAT } \\
\text { Mean } \pm \text { SD }\end{array}$ & $\begin{array}{c}\text { Hybrid } \\
\text { Mean } \pm \text { SD }\end{array}$ & $\begin{array}{l}\text { IMRT versus VMAT } \\
\quad p \text { value }\end{array}$ & $\begin{array}{l}\text { IMRT versus Hybrid } \\
\quad p \text { value }\end{array}$ & $\begin{array}{l}\text { VMAT versus Hybrid } \\
\quad p \text { value }\end{array}$ \\
\hline \multicolumn{7}{|l|}{ Normal lung } \\
\hline$D_{2 \%}(\mathrm{~Gy})$ & $67.5 \pm 2.7$ & $67.9 \pm 3.7$ & $67.7 \pm 3.2$ & $>0.05$ & $>0.05$ & $>0.05$ \\
\hline${ }^{*} V_{30}(\%)$ & $18.7 \pm 4.1$ & $18.4 \pm 4.2$ & $17.7 \pm 3.9$ & $>0.05$ & $<0.05$ & $<0.05$ \\
\hline${ }^{*} V_{20}(\%)$ & $25.4 \pm 4.9$ & $25.2 \pm 6.1$ & $25.5 \pm 5.6$ & $>0.05$ & $>0.05$ & $>0.05$ \\
\hline${ }^{*} V_{10}(\%)$ & $35.2 \pm 6.9$ & $41.7 \pm 8.0$ & $38.5 \pm 7.3$ & $<0.05$ & $<0.05$ & $<0.05$ \\
\hline${ }^{*} V_{5}(\%)$ & $50.0 \pm 8.2$ & $60.3 \pm 11.2$ & $57.2 \pm 10.7$ & $<0.05$ & $<0.05$ & $<0.05$ \\
\hline Mean (Gy) & $14.2 \pm 2.2$ & $15.2 \pm 2.6$ & $14.6 \pm 2.3$ & $<0.05$ & $<0.05$ & $<0.05$ \\
\hline \multicolumn{7}{|l|}{ Spinal cord } \\
\hline$D_{\max }(\mathrm{Gy})$ & $41.5 \pm 10.0$ & $35.7 \pm 10.5$ & $35.9 \pm 9.0$ & $<0.05$ & $<0.05$ & $>0.05$ \\
\hline \multicolumn{7}{|l|}{ Esophagus } \\
\hline$D_{\max }(\mathrm{Gy})$ & $67.0 \pm 5.7$ & $66.2 \pm 6.9$ & $63.7 \pm 7.9$ & $>0.05$ & $<0.05$ & $<0.05$ \\
\hline Mean (Gy) & $22.9 \pm 9.9$ & $23.0 \pm 9.6$ & $22.4 \pm 9.7$ & $>0.05$ & $<0.05$ & $<0.05$ \\
\hline \multicolumn{7}{|l|}{ Heart } \\
\hline$D_{2 \%}(\mathrm{~Gy})$ & $34.0 \pm 25.7$ & $31.3 \pm 24.0$ & $31.6 \pm 23.8$ & $>0.05$ & $<0.05$ & $>0.05$ \\
\hline Mean (Gy) & $8.5 \pm 8.8$ & $7.4 \pm 7.1$ & $7.8 \pm 7.8$ & $<0.05$ & $<0.05$ & $>0.05$ \\
\hline${ }^{*} V_{60}(\%)$ & $1.1 \pm 2.0$ & $0.9 \pm 1.6$ & $0.8 \pm 1.5$ & $<0.05$ & $<0.05$ & $>0.05$ \\
\hline${ }^{*} V_{40}(\%)$ & $4.5 \pm 5.6$ & $2.8 \pm 4.0$ & $3.2 \pm 4.4$ & $<0.05$ & $<0.05$ & $>0.05$ \\
\hline
\end{tabular}

PTV is planning target volume, IMRT is intensity modulated radiation therapy, VMAT is volumetric modulated arc therapy, CN is conformation number, and $\mathrm{HI}$ is homogeneity index.

${ }^{*} V_{N}$ is percentage volume of OARs receiving at least $N$ Gy of radiation dose.

3.2. Organs at Risk Sparing. The $V_{30}$ of normal lung for hybrid plans was significantly lower than IMRT plans $(17.7 \%$ versus $18.7 \% ; p<0.05)$ and VMAT plans $(17.7 \%$ versus $18.4 \% ; p<0.05)$. There was no significant difference in $V_{20}$ of normal lung among three techniques. The $V_{5}, V_{10}$, and mean lung dose (MLD) of normal lung for hybrid plans were $12.6 \%, 8.6 \%$, and $2.7 \%$ higher than those for IMRT plans, respectively $(p<0.05)$. However, the $V_{5}, V_{10}, V_{30}$, and MLD of normal lung for hybrid plans were $5.1 \%, 7.7 \%, 3.8 \%$, and $3.9 \%$ lower than those for VMAT plans, respectively $(p<0.05)$. The maximum doses of spinal cord and esophagus for hybrid plans were 5.6 Gy and 3.3 Gy lower than those for IMRT plans $(p<0.05)$. The mean doses of esophagus and heart for hybrid plans were $2.2 \%$ and $8.2 \%$ lower than IMRT plans $(p<0.05)$. The $V_{40}$ and $V_{60}$ of heart for hybrid plans were $27.3 \%$ and $28.9 \%$ lower than those for IMRT plans $(p<0.05)$.

3.3. Treatment Delivery Time and MUs. The mean delivery time of hybrid plans was longer than that of IMRT plans (327 s versus $280 \mathrm{~s} ; p<0.05$ ) and that of VMAT plans (327 s versus $114 \mathrm{~s} ; p<0.05)$. The number of mean MUs of hybrid plans $(797 \pm 81)$ was between the values of IMRT $(997 \pm 140)$ and VMAT plans $(509 \pm 53)$.

3.4. Dosimetric Evaluation Stratified by Target Volume. For the patients with the PTV volume smaller than $416.1 \mathrm{~cm}^{3}$, the mean $\mathrm{CN}$ was $0.72,0.86$, and 0.89 for IMRT, VMAT, and hybrid plans, respectively. Hybrid plans also significantly improved the PTV dose homogeneity compared with IMRT (9.9 versus 17.1; $p<0.05$ ) and VMAT (9.9 versus $14.9 ; p<$ $0.05)$. The mean $V_{5}$ and $V_{10}$ of normal lung for hybrid plans were $31.3 \%$ and $19.0 \%$, with an absolute difference of $4.1 \%$ and $1.1 \%$ lower than those for VMAT plans $(p<0.05)$, respectively. The MLD for hybrid plans was $6.8 \mathrm{~Gy}, 0.4 \mathrm{~Gy}$ lower than that for VMAT plans $(p<0.05)$. No difference of $V_{20}$ of normal lung among the IMRT, VMAT, and hybrid plans was found. The mean $V_{30}$ of normal lung for hybrid 
plans was $20.3 \%$ lower than that for IMRT plans $(p<0.05)$. No significant difference was found in the mean $V_{30}$ of normal lung between hybrid and VMAT plans. The maximum dose of spinal cord for hybrid plans was $27.6 \mathrm{~Gy}$, which was $4.3 \mathrm{~Gy}$ lower than that for IMRT plans $(p<0.05)$. The mean dose of esophagus for hybrid plans was $9.4 \mathrm{~Gy}$, which was $0.7 \mathrm{~Gy}$ lower than that for VMAT plans $(p<0.05)$. No differences in the mean $D_{\max }$ of esophagus and $D_{2 \%}$, mean dose, $V_{60}, V_{40}$ of heart among the IMRT, VMAT, and hybrid plans were found.

For the patients with the PTV volume larger than $416.1 \mathrm{~cm}^{3}$, the mean $\mathrm{CN}$ was $0.64,0.80$, and 0.83 for IMRT, VMAT, and hybrid plans, respectively. Hybrid plans also significantly improved the PTV dose homogeneity compared with IMRT (10.0 versus 14.6; $p<0.05$ ). The mean $V_{5}$ of normal lung for hybrid plans was $49.2 \%$, with an absolute difference of $4.4 \%$ lower than that for VMAT plans $(p<$ 0.05 ), while no difference was found for $V_{10}$ between two techniques. The MLD for hybrid plans was $10.4 \mathrm{~Gy}, 0.6 \mathrm{~Gy}$ lower than that for VMAT plans $(p<0.05)$. No differences of $V_{20}$ and $V_{30}$ of normal lung among the IMRT, VMAT, and hybrid plans were found. The maximum dose of spinal cord for hybrid plans was $37.0 \mathrm{~Gy}$, which was $5.8 \mathrm{~Gy}$ lower than that for IMRT plans $(p<0.05)$. No differences of $D_{\max }$, mean dose of esophagus and $D_{2 \%}$, mean dose, $V_{60}, V_{40}$ of heart among the IMRT, VMAT, and hybrid plans were found.

\section{Discussion}

In this study, we investigated a Hybrid IMRT/VMAT technique for primary nonsmall cell lung cancer. Compared with IMRT and VMAT, the improvements in conformity and homogeneity with Hybrid IMRT/VMAT were especially important when the target was in close proximity to the spinal cord limiting a satisfactory coverage of PTV. Compared with IMRT, Hybrid IMRT/VMAT significantly reduced the irradiated volume of the OARs and normal tissue receiving medium to high dose. Compared with VMAT, Hybrid IMRT/VMAT reduced the volume of normal lung receiving dose higher than $5 \mathrm{~Gy}, 10 \mathrm{~Gy}, 30 \mathrm{~Gy}$, and MLD significantly.

Several studies suggested that $V_{5}[11-14], V_{10}[12,14,15]$, and MLD $[14,15,17,18]$ were correlated with radiation pneumonitis, although the determination of the contributors to radiation pneumonitis was challenging, since a variety of treatment/patient-related factors appeared to influence this risk.

There were several studies demonstrating that VMAT could reduce delivery time and MUs compared with IMRT [12-15]. Reduction of delivery time could decrease the possibility of the intrafraction patient motion that leads to target underdosage and/or worse OARs sparing. However, the treatment delivery time of hybrid plans was longer than that of VMAT and IMRT plans in our study, because a hybrid plan comprised of both a 5-field IMRT and a 2 partial arcs VMAT. Liu et al. [19] reported that IMRT plans with fewer beams (five or seven beams) could achieve dosimetric quality comparable to those using nine equal-spaced beams, with reduced MUs and field segments. Using nine equal-spaced beams could allow more conformal plans but increased $V_{5}$ and $V_{10}$ of normal lung. So, we used 5-field IMRT plans to reduce the low dose distribution for normal lung. Chan et al. [20] reported that, in their pilot study of using VMAT, dosimetric distribution of one full arc was less favorable compared to those with two half arcs. So, 2 partial arcs VMAT was a good choice to compare with IMRT and Hybrid IMRT/VMAT.

Hybrid IMRT/VMAT improved the target dose conformity and homogeneity compared with IMRT and VMAT, while the difference of dose homogeneity of hybrid and VMAT plans became insignificant for the patients with the PTV volume larger than $416.1 \mathrm{~cm}^{3}$. The possible reason was that IMRT and VMAT made compromises in different aspects. IMRT achieved a reasonable dose distribution by intensity modulation with limited angular beam sampling. Due to the sparse angular sampling in IMRT, the conformity of the resultant dose distribution was often limited. On the other hand, while VMAT had sufficient angular sampling, it did not provide the desired intensity modulation in some beam directions. The final dose distribution depended on the level of intensity modulation and angular sampling. Hybrid IMRT/VMAT improved the target conformity and homogeneity by increasing the freedom to find the optimal combination of angular sampling and intensity modulation. The reason for the insignificance of homogeneity difference with increasing target volume between VMAT and hybrid plans was perhaps due to the fact that the homogeneity saturated by increasing the angular sampling in VMAT beyond a certain level, with the side effect of spreading low dose, which was also demonstrated as the reduced $V_{30}$ in VMAT and hybrid plans compared with IMRT for smaller targets, whereas no difference was found among three techniques for larger targets.

Several recent publications have introduced hybrid techniques which consisted of IMRT and arc with the purpose of combining the efficiency of arc and OARs sparing of IMRT. Martin et al. [17] reported that a novel IMRT \& Arc technique consisted of 4 -field IMRT in conjunction with a conformal arc. They demonstrated that for patients with esophageal cancers the IMRT \& Arc technique could potentially improve the therapeutic ratio in reduction of cardiorelated and pulmonary toxicity compared with plans for either helical tomotherapy or single-arc RapidArc plans. The forward planning for the conformal arc, as well as the manual IMRT beam arrangement, was used in their study. Similarly, Robar and Thomas [18] reported a HybridArc technique combining optimized dynamic conformal arcs and IMRT. In contrast to VMAT component in Hybrid IMRT/VMAT, the arc component of IMRT \& Arc and HybridArc did not involve intensity modulation, for example, via dose rate or gantry speed modulation, overlapping multiple arcs, or associated linac functionality. Compared with Hybrid IMRT/VMAT technique, the degrees of freedom of IMRT \& Arc and HybridArc were limited by (1) only a single pass by each arc, (2) constant dose rate, and (3) constant gantry speed. So no improvements in the brainstem and optic chiasm sparing were found in HybridArc compared with IMRT for the complex cranial cases. Chan et al. [20] reported that the Hybrid-RapidArc technique utilizing two arcs with additional static conformal fields could produce lower $V_{5}$, 
$V_{10}$, and MLD than double arcs RapidArc technique for lung cancers. However, Hybrid-RapidArc failed to meet the plan acceptance criteria due to the limited ability of intensity modulation with the conformal radiotherapy component, especially for the challenging cases (highly irregular PTV), with involving mediastinal lymphadenopathy. Furthermore, the ability to reduce the volume of normal lung receiving low doses was limited, because the intensity of the static beams could not be modulated to achieve good target conformity.

We developed a Hybrid IMRT/VMAT technique using IMRT as the base plan and then optimized the VMAT plan achieving trade-off between better dosimetric quality of IMRT and delivery efficiency (fewer MUs) of VMAT. This technique can be used on any treatment planning system capable of producing both VMAT and IMRT plans. Additional research work on the Hybrid IMRT/VMAT strategy is warranted in several areas. Most notable is to develop an optimization algorithm which can optimize both VMAT and IMRT simultaneously to determine the optimal proportion of the prescribed dose for the IMRT and VMAT components, the delivery sequence integrating the IMRT and VMAT components. Furthermore, the types of cancer sites and geometries that will benefit most from this Hybrid IMRT/VMAT technique should be further investigated.

We investigated the influence of prescription dose ratio between IMRT and VMAT in Hybrid IMRT/VMAT on the dose distribution and delivery efficiency, by creating the plans with the weighting of IMRT to VMAT of $1: 1,1: 2$, and $2: 1$. The results demonstrated that better conformity, homogeneity, sparing of normal lung from higher dose irradiation, and delivery efficiency were obtained with the increasing weight of the VMAT, with the cost of increasing the volume of low dose to normal lung $\left(V_{5}, V_{10}\right)$ and MLD. In addition, the ideal number of IMRT beams and VMAT arcs and the start and stop angle of arcs in hybrid plans would likely vary for different cases. For the representative case in this study, the beam angles of the IMRT plan were optimized using the beam angle optimization algorithm. Two right-anterior oblique fields and a right-posterior oblique field with gantry angles of $342^{\circ}, 306^{\circ}$, and $210^{\circ}$, a left-anterior oblique field with gantry angle of $39^{\circ}$, and a left-lateral field with gantry angle of $150^{\circ}$ were used. For the VMAT plan, two half arcs with the gantry angle $181^{\circ}$ to $0^{\circ}$ and $0^{\circ}$ to $181^{\circ}$ were used. We will further investigate a feasibility of automatic determination of these parameters for the individual patients in the optimization, so that the full potential of hybrid technique can be explored and the hybrid plans can be planned and delivered together, not separately. Hoover et al. [21] investigated an optimization and delivery technique called united intensity-modulated arc therapy (UIMAT), which optimized IMRT and VMAT simultaneously and delivered IMRT and VMAT in the same arc. They found that UIMAT has the potential to be superior to IMRT or VMAT.

The Hybrid IMRT/VMAT technique can be implemented to find the optimal compromise between gantry-angle and intensity modulation degrees of freedom, dosimetric quality, and delivery efficiency. It may be delivered without switching between delivery techniques in the future. That is, hybrid plans will be delivered as modulated arcs with IMRT inside, that is, IMRT control points (with no gantry motion) within a VMAT control point sequence (with gantry changes) rather than current two separate components, so that the delivery time would be further reduced. In addition, the emergence of autofield sequencing, which eliminates the unnecessary operator manual control of gantry rotation during dose delivery, and the dramatically increased dose rate in modern digital LINACs will make Hybrid IMRT/VMAT more efficient.

Our previous study demonstrated that some gantry angles benefited plan quality the most from beam modulation for some specific targets and OARs configuration [22]. Li and Xing [23] and Matuszak et al. [24] also demonstrated that an additional modulation from "optimal" beam angle improved plan quality compared with VMAT alone. While there were some optimal beam orientations that would benefit from IMRT, the selection of the best beam orientations for modulation might become increasingly difficult for the complicated cases. Li and Xing [23] proposed a dense angularly sampled and sparse intensity modulated RT (DASSIM-RT) strategy, in which a large number of beam angles were used to increase the angular sampling while simplifying the intensity modulation by eliminating the dispensable segments, to improve dose distribution while maintaining high delivery efficiency. In contrast with Hybrid IMRT/VMAT, DASSIMRT utilized an IMRT delivery mode, which could be time intensive. In addition, the number of beams and intensity level were arbitrarily selected in DASSIM-RT. Matuszak et al. [24] reported a similar strategy called FusionArc and proposed and validated gradient factor as the metric to find the optimal IMRT beam directions. They used a single-arc VMAT plan as the baseline plan and then converted selected VMAT apertures with the highest gradient into IMRT beams. Different from the arbitrarily selecting the number of beams and intensity level in DASSIM-RT, and using one arc and sequentially converting IMRT beam one by one in FusionArc, in our study, the Hybrid IMRT/VMAT integrated 5 IMRT fields and 2 partial VMAT arcs, in which the optimal IMRT beam directions were created by using beam angle optimization, while the VMAT arcs were optimized with the IMRT part as a base plan.

\section{Conclusions}

In combining VMAT and IMRT beams, Hybrid IMRT/ VMAT significantly improved both the target dose conformity and the homogeneity compared with IMRT and VMAT for nonsmall cell lung cancer. It reduced $V_{5}, V_{10}, V_{30}$, and MLD of normal lung compared with VMAT and protected the OARs better with fewer MUs with the cost of a little higher $V_{5}, V_{10}$, and mean lung dose (MLD) of normal lung compared with IMRT. Hybrid IMRT/VMAT technique can be a viable radiotherapy technique with better plan quality.

\section{Conflict of Interests}

This study was funded by the grant project: National Natural Science Foundation of China (no. 81071237). 


\section{References}

[1] R. R. Patel and M. Mehta, "Three-dimensional conformal radiotherapy for lung cancer: promises and pitfalls," Current Oncology Reports, vol. 4, no. 4, pp. 347-353, 2002.

[2] H. Murshed, H. H. Liu, Z. Liao et al., "Dose and volume reduction for normal lung using intensity-modulated radiotherapy for advanced-stage non-small-cell lung cancer," International Journal of Radiation Oncology Biology Physics, vol. 58, no. 4, pp. 1258-1267, 2004.

[3] W. Dörr and T. Herrmann, "Second primary tumors after radiotherapy for malignancies. Treatment-related parameters," Strahlentherapie und Onkologie, vol. 178, no. 7, pp. 357-362, 2002.

[4] E. J. Hall, "Intensity-modulated radiation therapy, protons, and the risk of second cancers," International Journal of Radiation Oncology, Biology, Physics, vol. 65, pp. 1-7, 2006.

[5] M. Pasler, D. Georg, S. Bartelt, and J. Lutterbach, "Node-positive left-sided breast cancer: does VMAT improve treatment plan quality with respect to IMRT?" Strahlentherapie und Onkologie, vol. 189, no. 5, pp. 380-386, 2013.

[6] A. Holt, D. Van Gestel, M. P. Arends et al., "Multi-institutional comparison of volumetric modulated arc therapy vs. intensitymodulated radiation therapy for head-and-neck cancer: a planning study," Radiation Oncology, vol. 8, article 26, 2013.

[7] S. D. McGrath, M. M. Matuszak, D. Yan, L. L. Kestin, A. A. Martinez, and I. S. Grills, "Volumetric modulated arc therapy for delivery of hypofractionated stereotactic lung radiotherapy: a dosimetric and treatment efficiency analysis," Radiotherapy and Oncology, vol. 95, no. 2, pp. 153-157, 2010.

[8] C. L. Ong, W. F. A. R. Verbakel, J. P. Cuijpers, B. J. Slotman, F. J. Lagerwaard, and S. Senan, "Stereotactic radiotherapy for peripheral lung tumors: a comparison of volumetric modulated arc therapy with 3 other delivery techniques," Radiotherapy and Oncology, vol. 97, no. 3, pp. 437-442, 2010.

[9] M. Rao, W. Yang, F. Chen et al., "Comparison of Elekta VMAT with helical tomotherapy and fixed field IMRT: plan quality, delivery efficiency and accuracy," Medical Physics, vol. 37, no. 3, pp. 1350-1359, 2010.

[10] X. Jiang, T. Li, Y. Liu et al., "Planning analysis for locally advanced lung cancer: dosimetric and efficiency comparisons between intensity-modulated radiotherapy (IMRT), singlearc/partial-arc volumetric modulated arc therapy (SA/PAVMAT)," Radiation Oncology, vol. 6, article 140, 2011.

[11] A. Van Riet, A. C. A. Mak, M. A. Moerland, L. H. Elders, and W. Van Der Zee, "A conformation number to quantify the degree of conformality in brachytherapy and external beam irradiation: application to the prostate," International Journal of Radiation Oncology Biology Physics, vol. 37, no. 3, pp. 731-736, 1997.

[12] A. S. Abbas, D. Moseley, Z. Kassam, S. M. Kim, and C. Cho, "Volumetric-modulated arc therapy for the treatment of a large planning target volume in thoracic esophageal cancer," Journal of Applied Clinical Medical Physics, vol. 14, no. 3, pp. 192-202, 2013.

[13] K. Nguyen, D. Cummings, V. C. Lanza et al., "A dosimetric comparative study: volumetric modulated arc therapy vs intensitymodulated radiation therapy in the treatment of nasal cavity carcinomas," Medical Dosimetry, vol. 38, no. 3, pp. 225-232, 2013.

[14] T.-F. Lee, P.-J. Chao, H.-M. Ting et al., "Comparative analysis of SmartArc-based dual arc volumetric-modulated arc radiotherapy (VMAT) versus intensity-modulated radiotherapy (IMRT) for nasopharyngeal carcinoma," Journal of Applied Clinical Medical Physics, vol. 12, no. 4, article 3587, 2011.

[15] M. T. Studenski, V. Bar-Ad, J. Siglin et al., "Clinical experience transitioning from IMRT to VMAT for head and neck cancer," Medical Dosimetry, vol. 38, no. 2, pp. 171-175, 2013.

[16] A. Doses, "3. Special considerations regarding absorbed-dose and dose-volume prescribing and reporting in IMRT," Journal of the ICRU, vol. 10, no. 1, pp. 27-40, 2010.

[17] S. Martin, J. Z. Chen, A. Rashid Dar, and S. Yartsev, "Dosimetric comparison of helical tomotherapy, RapidArc, and a novel IMRT \& Arc technique for esophageal carcinoma," Radiotherapy and Oncology, vol. 101, no. 3, pp. 431-437, 2011.

[18] J. L. Robar and C. Thomas, "HybridArc: a novel radiation therapy technique combining optimized dynamic arcs and intensity modulation," Medical Dosimetry, vol. 37, no. 4, pp. 358368, 2012.

[19] H. H. Liu, M. Jauregui, X. Zhang, X. Wang, L. Dong, and R. Mohan, "Beam angle optimization and reduction for intensitymodulated radiation therapy of non-small-cell lung cancers," International Journal of Radiation Oncology Biology Physics, vol. 65, no. 2, pp. 561-572, 2006.

[20] O. S. H. Chan, M. C. H. Lee, A. W. M. Hung, A. T. Y. Chang, R. M. W. Yeung, and A. W. M. Lee, "The superiority of hybrid-volumetric arc therapy (VMAT) technique over double arcs VMAT and 3D-conformal technique in the treatment of locally advanced non-small cell lung cancer-a planning study," Radiotherapy and Oncology, vol. 101, no. 2, pp. 298-302, 2011.

[21] D. A. Hoover, M. MacFarlane, E. Wong, J. J. Battista, and J. Z. Chen, "Feasibility of a unified approach to intensity-modulated radiation therapy and volume-modulated arc therapy optimization and delivery," Medical Physics, vol. 42, no. 2, pp. 726-734, 2015.

[22] R. Yang, J. Dai, Y. Yang, and Y. Hu, "Beam orientation optimization for intensity-modulated radiation therapy using mixed integer programming," Physics in Medicine and Biology, vol. 51, pp. 3653-3666, 2006.

[23] R. Li and L. Xing, "Bridging the gap between IMRT and VMAT: dense angularly sampled and sparse intensity modulated radiation therapy," Medical Physics, vol. 38, pp. 4912-4919, 2011.

[24] M. M. Matuszak, J. M. Steers, T. Long et al., "FusionArc optimization: a hybrid volumetric modulated arc therapy (VMAT) and intensity modulated radiation therapy (IMRT) planning strategy," Medical Physics, vol. 40, no. 7, Article ID 071713, 2013. 


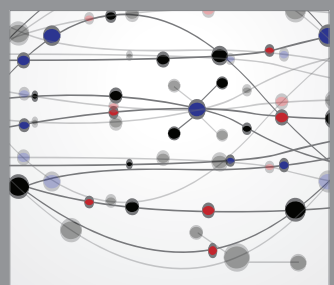

The Scientific World Journal
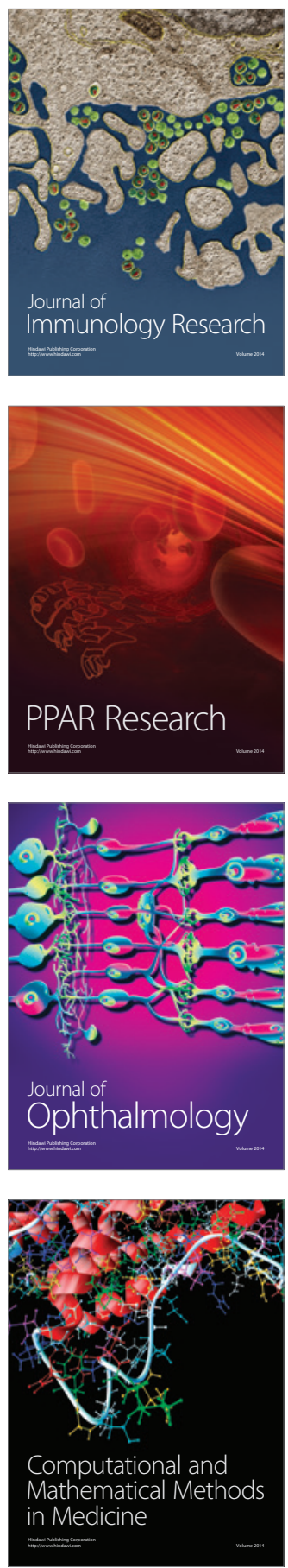

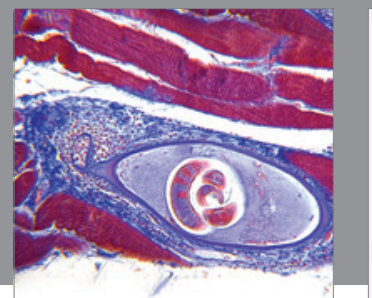

Gastroenterology

Research and Practice
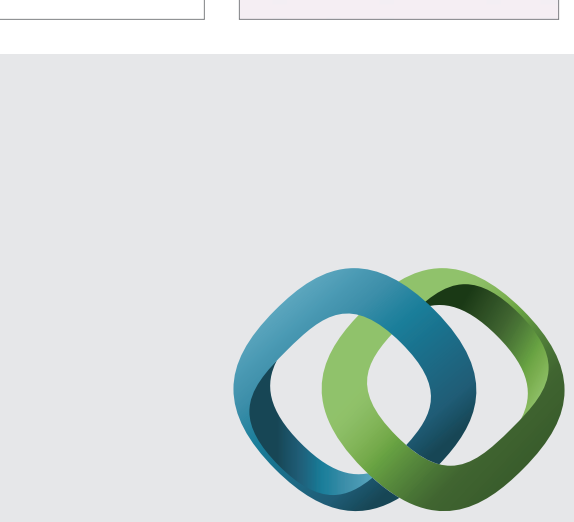

\section{Hindawi}

Submit your manuscripts at

http://www.hindawi.com
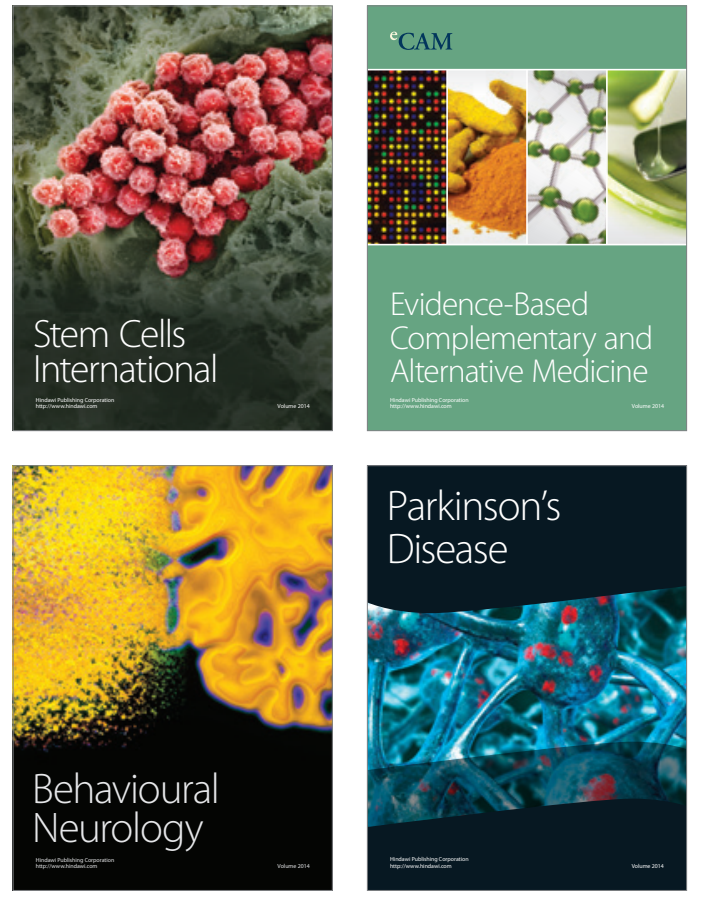
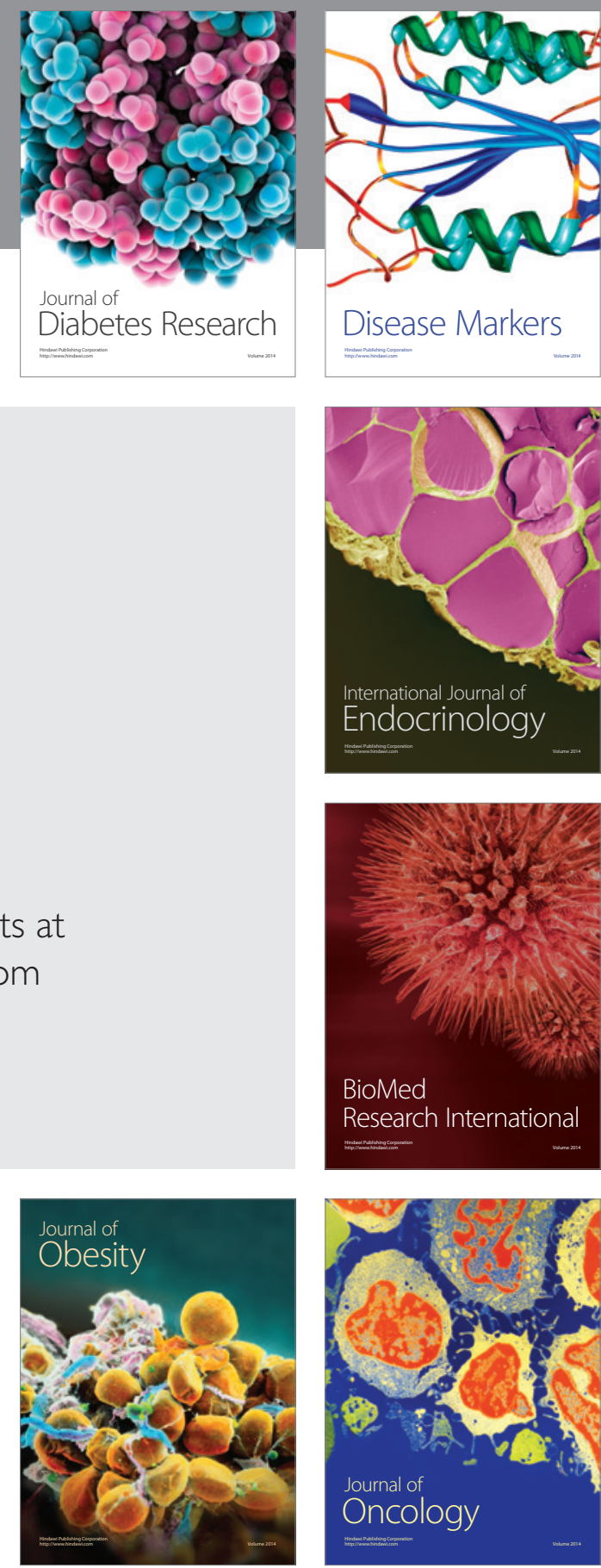

Disease Markers
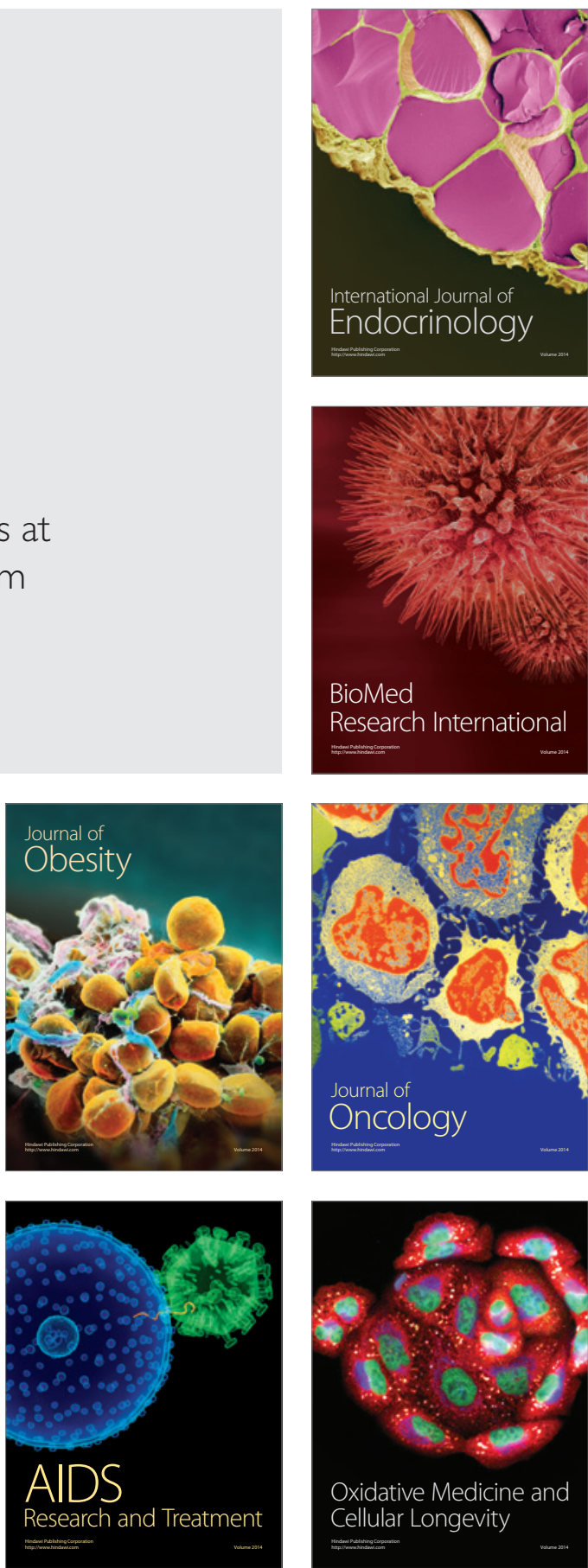